\title{
Interaction between DNA and Cationic Surfactants: Effect of DNA Conformation and Surfactant Headgroup ${ }^{\dagger}$
}

\author{
Rita S. Dias, ${ }^{*, *,}$ Luís M. Magno, ${ }^{\S}$ Artur J. M. Valente, ${ }^{\S}$ Dibyendu Das, ${ }^{\Perp}$ Prasanta K. Das, ${ }^{*, \|}$ \\ Souvik Maiti, ${ }^{*, \perp}$ Maria G. Miguel, ${ }^{\S}$ and Björn Lindman,$\S$ \\ Physical Chemistry 1, Centre for Chemistry and Chemical Engineering, University of Lund, \\ S-221 00 Lund, Sweden, Departamento de Química, Universidade de Coimbra, Rua Larga, \\ 3004-535 Coimbra, Portugal, Department of Biological Chemistry, Centre for Advanced Materials, Indian \\ Association for the Cultivation of Science, Jadavpur, Kolkata - 700032, India, and Institute for Genomics and \\ Integrative Biology, CSIR, Mall Road, Delhi 110007
}

Received: April 1, 2008; Revised Manuscript Received: July 25, 2008

\begin{abstract}
The interactions between DNA and a number of different cationic surfactants, differing in headgroup polarity, were investigated by electric conductivity measurements and fluorescence microscopy. It was observed that, the critical association concentration (cac), characterizing the onset of surfactant binding to DNA, does not vary significantly with the architecture of the headgroup. However, comparing with the critical micelle concentration (cmc) in the absence of DNA, it can be inferred that the micelles of a surfactant with a simple quaternary ammonium headgroup are much more stabilized by the presence of DNA than those of surfactants with hydroxylated headgroups. In line with previous studies of polymer-surfactant association, the cac does not vary significantly with either the DNA concentration or its chain length. On the other hand, a novel observation is that the cac is much lower when DNA is denaturated and in the single-stranded conformation, than for the double-helix DNA. This is contrary to expectation for a simple electrostatically driven association. Thus previous studies of polyelectrolytesurfactant systems have shown that the cac decreases strongly with increasing linear charge density of the polyion. Since double-stranded DNA (dsDNA) has twice as large linear charge density as single-stranded DNA (ssDNA), the stronger binding in the latter case indicates an important role of nonelectrostatic effects. Both a higher flexibility of ssDNA and a higher hydrophobicity due to the exposed bases are found to play a role, with the hydrophobic interaction argued to be more important. The significance of hydrophobic DNA-surfactant interaction is in line with other observations. The significance of nonelectrostatic effects is also indicated in significant differences in cac between different surfactants for ssDNA but not for dsDNA. For lower concentrations of DNA, the conductivity measurements presented an "anomalous" feature, i.e., a second inflection point for surfactant concentrations below the cac; this feature was not displayed at higher concentrations of DNA. The effect is attributed to the presence of a mixture of ss- and dsDNA molecules. Thus the stability of dsDNA is dependent on a certain ion atmosphere; at lower ion concentrations the electrostatic repulsions between the DNA strands become too strong compared to the attractive interactions, and there is a dissociation into the individual strands. Fluorescence microscopy studies, performed at much lower DNA concentrations, demonstrated a transformation of dsDNA from an extended "coil" state to a compact "globule" condition, with a broad concentration region of coexistence of coils and globules. The onset of DNA compaction coincides roughly with the cac values obtained from conductivity measurements. This is in line with the observed independence of cac on the DNA concentration, together with the assumption that the onset of binding corresponds to an initiation of DNA compaction. No major changes in either the onset of compaction or complete compaction were observed as the surfactant headgroup was made more polar.
\end{abstract}

\section{Introduction}

The interaction between DNA and cationic surfactants and lipids has received, since early times, a great interest from the biomedical sciences. More recently, physical chemists have devoted particular attention to these systems in an attempt to better understand the driving forces behind the molecular interactions; this is also expected to increase the efficiency and

\footnotetext{
$\dagger$ Part of the "Janos H. Fendler Memorial Issue".

* To whom correspondence should be addressed. E-mail: rsdias@qui.uc.pt, Fax: +351 239827703 (R.S.D.); E-mail: bcpkd@iacs.res.in, Fax: +91 33 24732805 (P.K.D); E-mail: souvik@igib.res.in, Fax: +91 1127667471 (S.M).

* University of Lund.

\& Universidade de Coimbra.

"Indian Association for the Cultivation of Science.

${ }^{\perp}$ CSIR.
}

number of uses for these systems. The strong associative behavior displayed by mixed systems of DNA and a cationic surfactant is well-known and is related to most of its applications such as extraction, purification, and counting. ${ }^{1-4}$ Also, the selfassembly properties of surfactants and the variety of structures they can form in solution, such as normal and reversed micelles, microemulsions, bilayers, and vesicles, make these molecules potential vehicles for host molecules in applications such as transport and drug delivery. In fact, membrane mimetic chemistry, the area of research based on membrane-mediated processes in organized surfactant assemblies and molecular hosts, is still of great importance. .,6 $^{-1}$

Single-chain surfactants typically self-assemble into micelles from a quite well-defined concentration, the critical micelle concentration $(\mathrm{cmc})$. For ionic surfactants, the $\mathrm{cmc}$ is orders of 
magnitude higher than that for nonionics. The lower stability of ionic micelles is due to an accumulation of counterions in the vicinity of the micelles; this organization of counterions lowers the entropy and counteracts micelle formation. The effect is partially eliminated upon addition of electrolyte and with multivalent counterions; this gives an important stabilization of the micelles and a lowering of the cmc. A dramatic stabilization of the micelles results if the counterions are polyions. The onset of micelle formation in the presence of a polymer is characterized by the critical association concentration (cac). The association between surfactants and oppositely charged polyelectrolytes has attracted a large interest (see, for example, refs 7-10); it is, for example, well-established that the interaction is strengthened, and the cac decreases strongly with the linear charge density of the polyion, and also that it is roughly independent of polymer molecular weight and concentration.

Conductivity measurements have been successfully used for the determination of the cac of polymer-oppositely charged surfactant systems. ${ }^{11-15}$ Surprisingly, however, this technique has not been used, to our knowledge, to investigate the interactions between DNA and cationic surfactants. Techniques that have been used to determine the cac of DNA-surfactant systems include fluorescence spectroscopy,${ }^{16,17}$ potentiometric titrations, ${ }^{18}$ and DNA gel swelling experiments. ${ }^{19}$ Electric conductivity measurements provide more accurate measurements than potentiometric titrations and have a clear advantage over the fluorescence spectroscopy since it does not require the addition of fluorescent probes. Regarding the DNA gels, it is unclear whether the constraints suffered by the DNA inside the gel network affects the cac values. Fluorescence microscopy is a technique that has been successfully used to study the compaction of double-stranded DNA (dsDNA) by cationic surfactants. ${ }^{18,20-26}$ DNA molecules in solution present an extended (coil) conformation, exhibiting a relatively slow wormlike motion and an average contour length, $L$, of about $4.0 \mu \mathrm{m}$. For sufficiently high concentrations of surfactant, the DNA molecules undergo compaction. These states are often referred to as globules and present a larger mobility in solution and a long-axis length of less than $1.0 \mu \mathrm{m}$. For intermediate concentrations, the two populations coexist in solution. It has been suggested that the concentration of surfactant at which the DNA starts to collapse corresponds to the cac. ${ }^{18}$

In this work we were interested mainly in two aspects of DNA-cationic surfactant association. While it is quite well established how an increasing hydrophobicity of the nonpolar part of the surfactant influences association, the influence of changing the headgroup is not well documented. Furthermore, previous work has focused on double-helix DNA, while the interactions of surfactants with single-stranded DNA (ssDNA) has essentially not been studied. Still differences between DNA conformational states in their association to surfactants are expected to deepen our insight into interactions significantly. In this work we investigated the cac of different types of hydroxylated cationic surfactants, where the $\mathrm{CH}_{3}$ groups in the cetyltrimethylammonium bromide (CTAB) headgroup have been sequentially replaced by hydroxyethyl groups (surfactants $\mathrm{S} 1$ to $\mathrm{S} 3$ in Scheme 1). We also investigated differences of cac between ds- and ssDNA.

\section{Materials and Methods}

Materials. Synthetic surfactants, CTAB, $N$-hexadecyl- $N, N$ dimethyl- $N$-(2-hydroxyethyl)ammonium bromide (S1), $N$-hexadecyl- $N$-methyl- $N, N$-bis(2-hydroxyethyl)ammonium bromide (S2), and $N$-hexadecyl- $N, N, N$-tris(2-hydroxyethyl)ammonium chloride
SCHEME 1: Structure of the Surfactants Used in This Study ${ }^{a}$

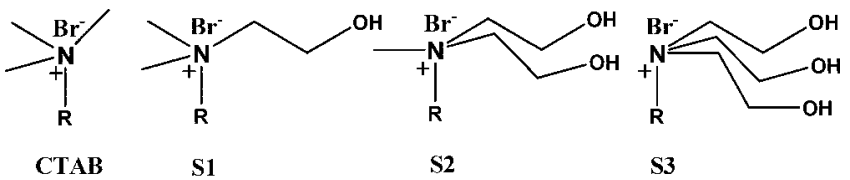

$\mathbf{R}=\mathrm{C}_{16} \mathrm{H}_{33}$

${ }^{a}$ The $-\mathrm{CH}_{3}$ groups of $\mathrm{CTAB}$ were sequentially replaced by hydroxyethyl groups in the surfactant headgroup (S1 to S3).

(S3) were synthesized according to procedures described in ref 27. Their structures are shown in Scheme 1.

For electrical conductivity measurements, salmon sperm DNA solutions were purchased from Invitrogen at $10 \mathrm{mg} / \mathrm{mL}\left(\mathrm{A}_{260}\right)$ and with a size range of $\leq 2000 \mathrm{bp}$ ( $1 \% \mathrm{TAE}$ agarose gel). For fluorescence microscopy studies, coliphage T4 DNA $(M=1.1$ $\times 10^{8} \mathrm{Da}$, ca. $167 \mathrm{kbp}$ ) was supplied by Wako Nippon Gene. The DNA concentrations were determined spectrophotometrically considering the molar extinction coefficient of DNA bases to be equal to $6600 \mathrm{M}^{-1} \mathrm{~cm}^{-1}$. ${ }^{28}$ The ratios of the absorbance at 260 and $280 \mathrm{~nm}$ of the stock solutions were found to be 1.8 and 1.9 for the two types of DNA. The fluorescence dyes, $4^{\prime}, 6-$ diamidino-2-phenylindole (DAPI) and Gelstar were obtained from Sigma and Cambrex, respectively. Ascorbic acid, the antioxidant, and Tris base were purchased from Sigma.

Sample Preparation. For the fluorescence microscopy measurements, the samples were prepared by diluting the DNA stock solutions into the buffer solution containing an antioxidant (ascorbic acid) and the fluorescent dye (DAPI or GelStar). The samples were left to equilibrate for $10 \mathrm{~min}$ and after that the appropriate volume of the surfactant stock solutions was added, and the samples were equilibrated for $30 \mathrm{~min}$ more. The final concentrations were as follows: DNA, in nucleotide units, 0.5 $\mu \mathrm{M}$, fluorescent dye $0.5 \mu \mathrm{M}$ (DAPI) or $1 \times$ (GelStar), and ascorbic acid $0.5 \mathrm{M}$. Under these conditions, the binding number of DAPI per $1 \mathrm{bp}$ of DNA, in an aqueous buffer solution, is estimated to be equal to 0.05 , and the persistence length of the DNA chain is expected to remain nearly the same as in the absence of dye..$^{29}$ All stock solutions were prepared with 10 $\mathrm{mM}$ Tris-HCl buffer ( $\mathrm{pH}$ 7.6). For the electrical conductivity measurements, the DNA and surfactant stock solutions were prepared in $2 \mathrm{mM}$ Tris-HCl buffer ( $\mathrm{pH}$ 7.6). This (lower) concentration was chosen so that the ionic strength of the solution would not mask the changes in conductivity due to the DNA-surfactant interaction. The ssDNA solutions were prepared by thermal denaturation of a DNA stock solution; the DNA solution was heated to $75{ }^{\circ} \mathrm{C}$ for $15 \mathrm{~min}$ and then immediately dipped into ice for fast cooling, to prevent renaturation.

Electrical Conductivity Measurements. Electrical resistances of the solutions were measured with a Wayne-Kerr model 4265 automatic LCR meter at $2 \mathrm{kHz}$. A dip-type conductance cell with a cell constant of $0.119 \mathrm{~cm}^{-1}$, uncertainty of $0.02 \%$, was used. Cell constants were measured using the procedure described elsewhere. ${ }^{30}$ Measurements were made at 25.00 $( \pm 0.01){ }^{\circ} \mathrm{C}$ in a Grant thermostat bath. In a typical experiment, $25 \mathrm{~mL}$ of the DNA solution was placed in the conductivity cell; then, aliquots of surfactant were added using a $25 \mu \mathrm{L}$ syringe.

Fluorescence Microscopy. The conformation map of DNA in the presence of surfactants was obtained as follows. The samples were placed on microscope glasses (No.0, Chance Propper, England) and were illuminated with a UV mercury lamp. The fluorescence images of single DNA molecules were 
observed using a Zeiss Axioplan microscope equipped with a $100 \times$ oil-immersed objective lens and were digitized on a personal computer through a high-sensitivity SIT C-video camera and an image processor, Argus-20 (Hamamatsu Photonics, Japan). The observations were carried out at $25^{\circ} \mathrm{C}$.

\section{Results and Discussion}

Remarks on DNA and Surfactant Self-Assembly. While the significance of the amphiphilic nature of surfactants is well appreciated, the amphiphilic nature of DNA is often overlooked. In fact DNA is an amphiphilic polymer and its self-assembly plays a ubiquitous role. We, therefore, start our discussion with some general remarks on the self-assembly of surfactants, of DNA, as well as of mixed polyelectrolyte-surfactant systems, returning to them specifically in analyzing the experimental data below.

As indicated by the 2 orders of magnitude higher cmc's than for nonionic surfactants, there is a strong electrostatic opposing force for ionic surfactant micellization. This is entropic in nature and is due to the accumulation of counterions in the vicinity of the highly charged micelles. The cmc of ionic surfactants decreases by a factor of 2 upon lengthening the alkyl chain by one methylene group; for nonionics, the factor is instead 3, this difference having the same electrostatic origin as the difference in cmc values. ${ }^{31}$

The self-assembly of surfactants is also dependent on the size of the headgroup. However, as exemplified by nonionic ethyleneoxide (EO) surfactants, the effect is weak on the cmc but strong on the type of aggregate formed. For example, for dodecyl surfactants, the cmc's differ only moderately as the number of EO groups changes from 5 to 8; the micelles are small and spherical with 8 EOs and long wormlike with 5 EOs.

The effect of headgroup size on self-assembly structure is fruitfully discussed on the basis of the critical packing parameter. For ionic surfactants, the appropriate headgroup size must include the effective electrostatic repulsion between head-groups. As discussed below, some of the ionic surfactants investigated in this work have bulky head-groups, and the effective headgroup size will contain both steric and electrostatic components; the latter but not the former will be sensitive to the ionic environment.

A DNA molecule contains bases, which are hydrophobic, and carbohydrate and phosphate groups, which are hydrophilic. The hydrophobic parts promote an association between DNA molecules, while the hydrophilic ones, in particular the ionic groups, oppose self-assembly. The most important self-assembly structure of DNA is the double-helix. The delicate balance between opposing forces is best illustrated by the fact that, in the absence of electrolyte, the double-helix becomes unstable and dissociates into two single strands. Such a dissociation or denaturation is also observed as temperature is increased. ${ }^{32}$

Cationic surfactants also affect the stability of the doublehelix. However, depending on alkyl chain length, there can be either a stabilization or a destabilization. This is due to the interplay between electrostatic interactions, favoring the double helix, and hydrophobic interactions, favoring the single strands, having more exposed bases.

If an ionic surfactant is added to a solution of a polyelectrolyte, a strongly cooperative association can be inferred. It is best described as a polymer-induced surfactant self-assembly and is due to the strong increase in the counterion entropy since many monovalent counterions are replaced by a polyion. The cmc of a surfactant in the presence of a polyelectrolyte, cac, can be lowered by orders of magnitude. ${ }^{33}$ Important for our packing considerations below is that in mixed polyelectrolytesurfactant systems, electrostatic effects are effectively quenched; this is shown by the fact that the cac decreases by a factor of 3 as one methylene group is added to the alkyl chain, the same figure as for nonionic surfactant micellization. A consequence in packing considerations is that the headgroup size is only dependent on steric effects.

The two features of DNA mentioned above deserve special attention in studies of its effect on surfactant self-assembly, i.e., its amphiphilicity and its self-assembly. Regarding the first point, it is well-documented that the presence of hydrophobic groups in a polymer decreases the cac; for strongly hydrophobic groups, a case not applicable here, the cooperative binding may turn into a noncooperative one. ${ }^{33}$ Regarding the second point, a surfactant will, as described, affect DNA self-assembly.

Electrical Conductivity. As mentioned above, we have used conductivity measurements to determine the cac of the different surfactants under study in the presence of DNA.

The specific electrical conductances, $\kappa$, of CTAB and S3 as a function of the molar concentration of the surfactant are plotted in Figure 1 (left panels). $\kappa$ is calculated from the experimental specific conductance, $\kappa_{\exp }$, and corrected for the specific conductance of the DNA solutions, $\kappa_{0}$, according to $\kappa=\kappa_{\exp }-$ $\kappa_{0}$. The cmc of the surfactants in the presence of DNA (cac) was computed using the second derivative of the specific conductance as a function of the surfactant concentration, as described in ref 34. This is also plotted in Figure 1, for the CTAB case.

The curves obtained are very similar to those of other polyelectrolyte-oppositely charged surfactant systems presented in the literature. The value obtained for the cac for the DNA-CTAB system was $4 \pm 1 \mu \mathrm{M}$. This is close to other values obtained for the same system using different techniques: $5 \mu \mathrm{M}$ and $10 \mu \mathrm{M}$ by fluorescence spectroscopy using YOYO$1^{16}$ and ethidium bromide, ${ }^{17}$ respectively; and $8 \mu \mathrm{M}$ by potentiometric titrations. ${ }^{18}$ For the case of the interaction of CTAB with DNA gels, the cac was determined to be $20 \mu \mathrm{M} ;{ }^{19}$ this somewhat higher value is probably due to the constraints felt by the polyelectrolyte in the gel network. As shown by Wallin and Linse, ${ }^{35}$ the polymer-surfactant association is weaker for polymer chains with lower conformational freedom.

The cac values for the other surfactants under study, also determined by conductivity, are presented in Table 1. As can be seen from the plots in the right-hand side of Figure 1 (note the difference in the axis) and the values presented in Table 1, all the surfactants present roughly the same cac values, independently of the geometry of the headgroup. It is interesting to note thus that the surfactant self-assembly in the presence of DNA does not vary between the quite different surfactant headgroups; this contrasts the dramatic changes observed when the alkyl chain length is varied. ${ }^{25,36}$

The invariance of the cac with changes in the headgroup contrasts two other observations: first, the cmc's of the surfactants are quite different, and, second, the accessibility of DNA to ethidium bromide binding is considerably higher for the more hydrophilic surfactants, as shown in a previous publication. ${ }^{27}$ However, both observations can easily be rationalized from our understanding of surfactant self-assembly. It is well-known that by adding a (even weak) nonionic amphiphile, such as an alcohol, ionic surfactant micelles are stabilized by an electrostatic screening effect, and thus the cmc is lowered; ${ }^{33}$ the lower cmc of the hydroxylated surfactants is ascribed to an analogous effect. In the presence of DNA, this effect is completely overshadowed by the stabilization effect by the polyion and, 

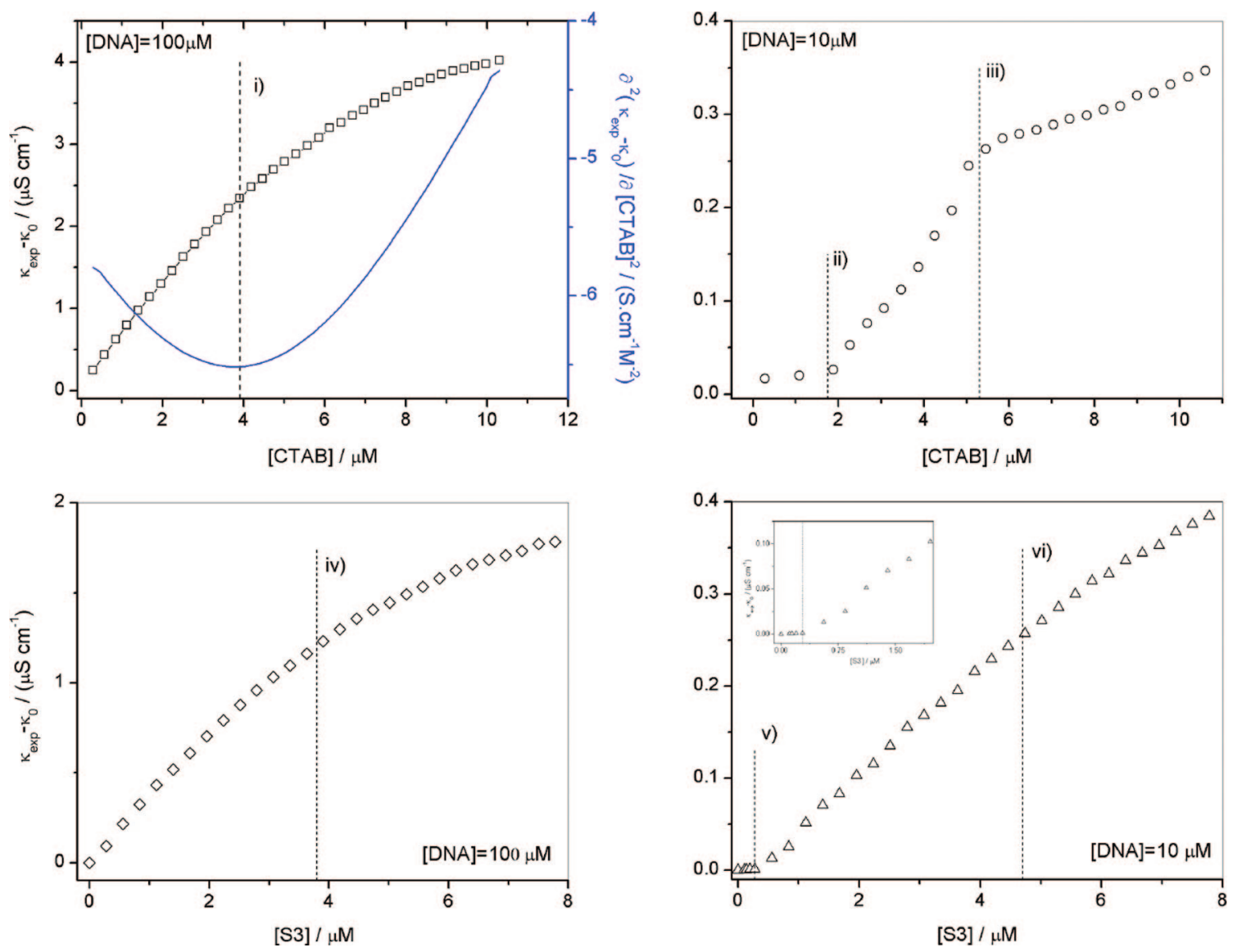

Figure 1. Specific conductances, $\kappa=\kappa_{\exp }-\kappa_{0}$, of CTAB (top) and S3 (bottom) in DNA solutions of different concentrations, 100 (left) and 10 (right) $\mu \mathrm{M}$. In the first graph, the second derivative used in the calculation of the cac is shown. Vertical dashed lines show inflection points: (i) $[\mathrm{CTAB}]=4( \pm 1) \mu \mathrm{M}$, (ii) $[\mathrm{CTAB}]=1.7( \pm 0.1) \mu \mathrm{M}$, (iii) $5( \pm 1) \mu \mathrm{M}$, (iv) $[\mathrm{S} 3]=3.8( \pm 0.5) \mu \mathrm{M}$, (v) $[\mathrm{S} 3]=0.28( \pm 0.05) \mu \mathrm{M}$, and $(\mathrm{vi})[\mathrm{S} 3]=$ $4.7( \pm 0.5) \mu \mathrm{M}$.

TABLE 1: Values of the cmc of the Different Surfactants, cac Determined Using Conductivity Data of the Different DNA - Surfactant Solutions at Two Different DNA Concentrations, and cac' $^{\prime}$ Observed for Systems Prepared with $10 \mu \mathrm{M}$ of DNA in Phosphate Groups ${ }^{a}$

\begin{tabular}{lccccc}
\hline & & DNA, $100 \mu \mathrm{M}$ & & \multicolumn{2}{c}{ DNA, $10 \mu \mathrm{M}$} \\
\cline { 5 - 6 } & $\mathrm{cmc}^{b} / \mathrm{mM}$ & $\mathrm{cac} / \mu \mathrm{M}$ & & $\mathrm{cac} / \mu \mathrm{M}$ & $\mathrm{cac}^{\prime} / \mu \mathrm{M}$ \\
\hline CTAB & 0.92 & $4( \pm 1)$ & & $5( \pm 1)$ & $1.7( \pm 0.5)$ \\
S1 & 0.20 & $4.33( \pm 0.04)$ & & $4( \pm 1)$ & $0.64( \pm 0.04)$ \\
S2 & 0.15 & $3.2( \pm 0.4)$ & & $4.8( \pm 0.5)$ & $0.42( \pm 0.06)$ \\
S3 & 0.04 & $3.8( \pm 0.5)$ & & $4.7( \pm 0.5)$ & $0.28( \pm 0.05)$
\end{tabular}

${ }^{a}$ The cac measurements were performed in a $2 \mathrm{mM}$ Tris- $\mathrm{HCl}$ buffer solution (pH 7.6) at $25^{\circ} \mathrm{C} .{ }^{b}$ Data from ref 27.

therefore, differences disappear. However, now another difference between the headgroups becomes accentuated, i.e., that of mere physical size; hydroxylation increases the headgroup volume considerably. It is well-established that surfactant packing determines the type of aggregate formed; basically, the larger the headgroup, the smaller the aggregates, ${ }^{27}$ leading to small spherical micelles for surfactants with large headgroups, while larger aggregates are obtained with small headgroups. For the surfactants studied alone, differences are small since the effective headgroup size is strongly determined by the electrostatic repulsions. However, as these are essentially eliminated in the complex with DNA, we expect CTAB to form large micelles, while the micelles become progressively smaller with increasing number of hydroxylated substituents. With smaller micelles, the polyion surface will be less completely covered, which allows for accessibility for the binding of a fluorescent probe such as ethidium bromide. Preliminary results using smallangle X-ray scattering have confirmed the loss of the hexagonal structure obtained for the CTAB with the hydroxylation of the surfactant headgroup (results not shown), which confirms the decrease in the size of the surfactant aggregates.

In order to investigate the dependence of the cac on the concentration of DNA, we have also performed conductivity experiments using DNA solutions with $10 \mu \mathrm{M}$, Figure 1 (right). While we did observe a cac close to that obtained at higher DNA concentrations, and thus confirming the general view that cac is independent of polymer concentration, additional observations were made. We observed an initial behavior of the conductivity, which, to the best of our knowledge, has not been reported before. Thus, surprisingly, the graph looks very different from the one obtained when using DNA concentrations of $100 \mu \mathrm{M}$ (left vs right-hand side plots in Figure 1). There is a deviation from linearity for very low concentrations of surfactant. The conductance increases with the first addition of the surfactant solution, as expected, but successive additions do not lead to a significant increase in the specific conductance. This behavior was observed for all the surfactants, and the concentration at which the first inflection point ( $\left.\mathrm{cac}^{\prime}\right)$ was observed is presented in Table 1. At higher concentrations of surfactant a second inflection point was observed, and this was determined to be the cac. The cac values are again very similar for all four surfactants. Also the values are very close to the ones obtained for higher concentrations of DNA suggesting, as 

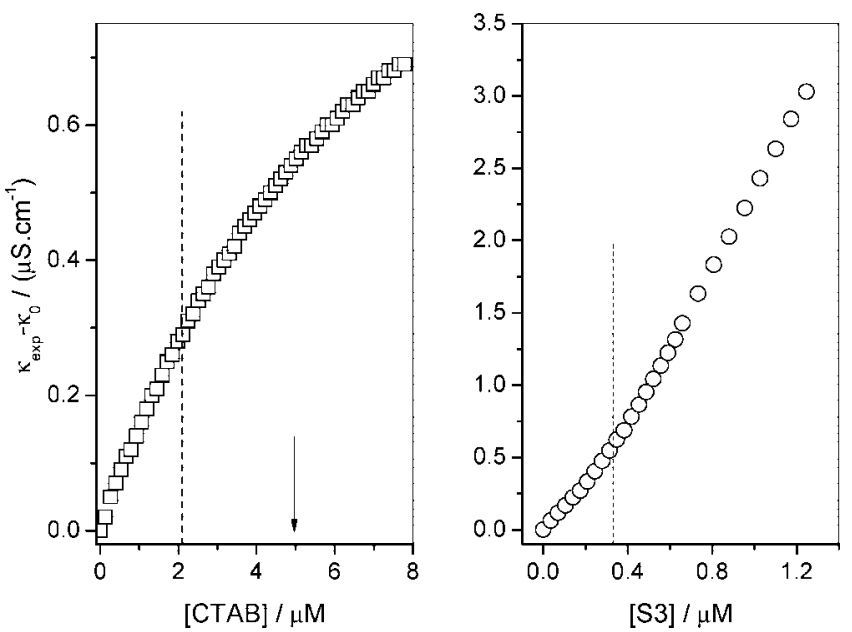

Figure 2. Specific conductances, $\kappa=\kappa_{\exp }-\kappa_{0}$, of CTAB (left) and S3 (right) in thermally denaturated DNA samples at $10 \mu \mathrm{M}$. Vertical dashed lines show cac for CTAB and S3, $2.1( \pm 0.2) \mu \mathrm{M}$ and 0.33 $( \pm 0.07) \mu \mathrm{M}$, respectively. The arrow shows the CTAB cac in the presence of dsDNA $10 \mu \mathrm{M}$.

said above, that the cac for these systems is independent of the DNA concentration.

Regarding this behavior, not observed for other polyelectrolytes, we attribute it to the special feature of DNA of a transition between double- and single-stranded forms. Thus we refer it to the above-mentioned amphiphilic nature of DNA, with the nonpolar bases driving a hydrophobic association of two DNA strands into the double helix. The delicate balance between hydrophobic attraction and electrostatic repulsion is illustrated by the fact that an electrostatic screening is required for the stabilization of dsDNA. Thus, in the absence of a sufficiently large ionic atmosphere, dsDNA dissociates into two separate strands. This ion concentration can be provided by the dissociated counterions, i.e., there is a self-screening effect. ${ }^{37-39} \mathrm{It}$ is thus our suggestion, since we are working with relatively low concentrations of DNA and solutions with low ionic strength, that there is a mixture of ssDNA and dsDNA molecules. It should be noted that the DNA we worked with in these experiments, salmon sperm DNA, is not well characterized in terms of chain length and base composition; therefore, the renaturation of fully dissociated dsDNA molecules is not likely. To check this hypothesis, we have performed conductivity experiments using thermally denaturated DNA solutions at 10 $\mu \mathrm{M}$ (Figure 2). For these systems, the curves are very similar to that of the DNA solution at $100 \mu \mathrm{M}$, presenting only one inflection point. The cac for the ssDNA-CTAB system was found to be $2.1 \pm 0.2 \mu \mathrm{M}$, and for the ssDNA-S3 system it was $0.33 \pm 0.07 \mu \mathrm{M}$, both of which are in very good agreement with the CTAB and S3 concentrations at which the first inflection was observed.

It is interesting to note that the cac for the ssDNA-CTAB system is about half of the cac of dsDNA-CTAB, and even lower for the S3 surfactant. A lower value of the cac for the ssDNA-CTAB system was also observed for covalent DNA gels (20 and $15 \mu \mathrm{M}$ of CTAB for ds- and ssDNA, respectively). ${ }^{40}$ A stronger interaction with oppositely charged surfactants for ssDNA than for dsDNA was also inferred in phase diagram studies. ${ }^{39}$

It is a general observation, as also suggested by theoretical considerations, that the interaction between a polyelectrolyte and an oppositely charged surfactant becomes stronger as the polyion linear charge density increases; this is inter alia borne

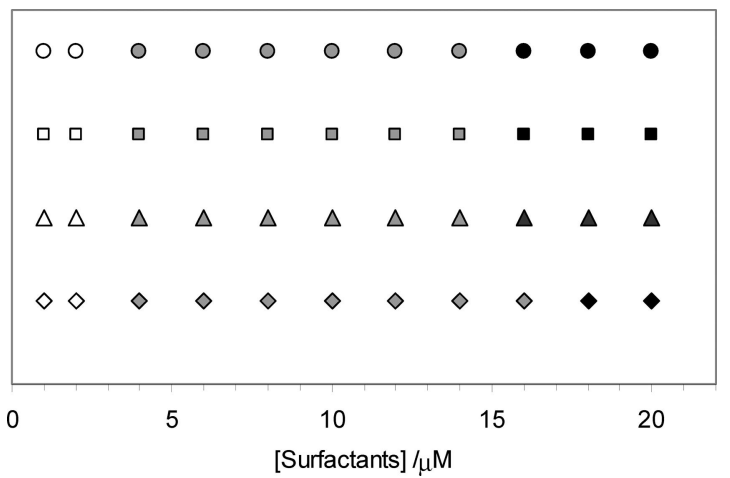

Figure 3. Conformational map of single T4 DNA molecules, $0.5 \mu \mathrm{M}$ in $10 \mathrm{mM}$ Tris- $\mathrm{HCl}$ buffer $(\mathrm{pH} 7.6)$ on the stepwise addition of CTAB $(\diamond)$, S1 $(\square)$, S2 $(\Delta)$, and S3 $(\bigcirc)$. Open symbols correspond to the extended conformation of DNA, filled symbols correspond to the globular DNA conformation, and shaded symbols correspond to the coexistence of the two populations. $T=25^{\circ} \mathrm{C}$.

out in cac values and in phase diagrams. ${ }^{41-44}$ Our observations are thus contrary to expectations. There are two possible explanations for why simple electrostatic considerations fail. First, as shown by Wallin and Linse, at a given charge density, the interaction between a linear polyion and a spherical macroion becomes stronger as the polyion flexibility increases; ${ }^{45}$ a flexible polyelectrolyte such as ssDNA would more easily wrap around a micelle and associate more closely than the rigid dsDNA. Second, in ssDNA, the hydrophobic bases are more exposed than in dsDNA, leading to a significant hydrophobic interaction between polyion and surfactant. While a definite conclusion is difficult to draw at present, there are strong indications, as we have discussed recently, ${ }^{46}$ that the latter contribution is most significant.

It is also interesting to note that the cac for ssDNA is different for the different surfactants considered, contrarily to the dsDNA systems, following now the same trend as the cmc; surfactants with higher $\mathrm{cmc}$ values also presented a higher cac. We interpret this in terms of nonelectrostatic contributions for the stability of the micelles. For dsDNA systems, the electrostatic interactions are, by far, the predominant and, as mentioned above, the differences between the surfactants are overshadowed by the presence of the DNA. For the ssDNA systems, nonelectrostatic contributions, as the ones described above, play an important role.

Fluorescence Microscopy. To confirm the cac values using a second method, we have used fluorescence microscopy. In Figure 3 is represented the conformational map for the DNA molecules in the presence of the four surfactants.

The open symbols correspond to DNA molecules that are in the coil (or extended) conformation, the filled symbols correspond to DNA molecules that have a sufficient amount of surfactant bound to them as to induce their collapse, and the samples at which the two populations coexist are represented with shaded symbols. The diamonds show the conformational map of DNA in the presence of CTAB. For this particular system, the coexistence region starts for surfactant concentrations of around $4 \mu \mathrm{M}$ and ends at $18 \mu \mathrm{M}$. These values are slightly different from those reported for CTAB in a previous publication. ${ }^{25}$ This we attribute to the fact that we have used ascorbic acid instead of mercaptoethanol as antioxidant. Presumably the ascorbic acid will have a smaller influence and, in fact, the results obtained here are very similar to those obtained by dynamic light scattering experiments, where the experiments were conducted without the addition of fluorescence dye or 
antioxidants. ${ }^{47}$ Experiments were also performed using either DAPI or GelStar as fluorescence dyes. Since the results were identical for the two dyes, we continued the experiments using GelStar only, since it gave a better contrast in these experiments and was, therefore, easier to work with.

In the same graph are also presented the results for the surfactants with the hydroxyl substituents at the headgroup. As can be observed, the concentrations at which the surfactants start to compact dsDNA as well as the end of the coexistence region are roughly the same for the four surfactants. This indicates that the differences in the headgroup do not influence substantially the compaction of DNA.

The compaction of large DNA molecules is driven by ioncorrelation effects, ${ }^{48}$ that is, the effective attraction between two highly charged surfaces (or different parts of the molecule in this case) that arises as a result of the presence of multivalent ions ${ }^{49}$ or in solutions with low dielectric constants. ${ }^{50}$ In the case of monovalent cationic surfactants, such as the ones used in this study, the attraction arises from the formation of surfactant aggregates in the vicinity of the DNA, at the cac; the surfactant aggregates act as multivalent counterions with concomitant ion correlation effects. The cac values obtained for the beginning of the compaction region and the cac's measured by electric conductivity are in good agreement.

It should finally be noted that the concentration of DNA in the two different types of experiments is very different as well as the size of the molecules (167 vs $2 \mathrm{kbp}$ ). This suggests that the cac is also independent of the molecular weight of the polyelectrolyte, as found also in other studies of surfactant binding to oppositely charged polyelectrolytes.

\section{Conclusions}

In this paper we have described the interactions between DNA and cationic surfactants of different headgroup architectures based on studies by electric conductivity measurements and fluorescence microscopy. It was observed that, even though the cmc of the surfactants decreases with the headgroup size, the cac does not vary significantly with the architecture of the headgroup, which indicates that the degree of stabilization of the micelles in the presence of DNA is very different; in the polyion-surfactant complexes electrostatic contributions to the micellization are thus found to be eliminated.

The cac does not vary significantly with the DNA concentration and chain length. It is interesting to note, however, that the cac is much lower when DNA is in the single-stranded conformation than the double-stranded conformation, even though the dsDNA presents a much higher charge density. This and the fact that the cac is dependent on the architecture of the surfactant headgroup for ssDNA- surfactant systems, points to nonelectrostatic contributions to the stability of the surfactant micelles, possibly the higher flexibility and/or hydrophobicity of the ssDNA when compared to the double-stranded counterpart. For certain experimental conditions we have observed two cac values for the studied systems, which we attribute to a mixture of ss- and dsDNA molecules.

Fluorescence microscopy studies showed an excellent agreement with the conductivity measurements; the coexistence of coils and globules was observed for roughly the same concentration as the cac, and it was the same for all four surfactants. Also the coexistence region width was roughly the same for all four surfactants under study.

Acknowledgment. R.S.D, M.G.M., and B.L. acknowledge Fundação para a Ciência e Tecnologia, Portugal (Grant SFRH/
BPD/24203/2005 and Projects PTDC/QUI/67962/2006 and POCTI/QUI/58689/2004). The authors acknowledge the Swedish International Development Cooperation Agency (SIDA) for financial support.

\section{References and Notes}

(1) Jost, J. P.; Jiricny, J.; Saluz, H. Nucleic Acids Res. 1989, 17, 21432143.

(2) Delsal, G.; Manfioletti, G.; Schneider, C. Biotechniques 1989, 7, 514-519.

(3) Ishaq, M.; Wolf, B.; Ritter, C. Biotechniques 1990, 9, 19-24.

(4) Sibatani, A. Anal. Biochem. 1970, 33, 279-285.

(5) Fendler, J. H. Membrane Mimetic Chemistry - Characterizations and Applications of Micelles, Microemulsions, Monolalyers, Bilayers, Vesicles, Host-Guest Systems and Polyions; John Wiley: New York, 1982.

(6) Fendler, J. H. Аnnu. Rev. Phys. Chem. 1984, 35, 137-157.

(7) Polymer-Surfactant Systems; Kwak, J. C. T., Ed.; Marcel Dekker: New York, 1998, p 482.

(8) Lindman, B.; Thalberg, K. In Interaction of Surfactants with Polymers and Proteins; Goddard, E., Ananthapadmanabhan, K., Eds.; CRC Press: Boca Raton, FL, 1993; pp 203-276.

(9) Dias, R. S.; Lindman, B.; Miguel, M. G. J. Phys. Chem. B 2002, 106, 12608-12612.

(10) Hansson, P.; Lindman, B. Curr. Opin. Colloid Interface Sci. 1996, 1, 604-613.

(11) Kogej, K.; Skerjanc, J. Langmuir 1999, 15, 4251-4258.

(12) Tomasic, V.; Tomasic, A.; Filipovic-Vincekovic, N. J. Colloid Interface Sci. 2002, 256, 462-471.

(13) Bakshi, M. S.; Sachar, S. Colloid Polym. Sci. 2004, 282, 993-999.

(14) Tomasic, V.; Tomasic, A.; Smit, I.; Filipovic-Vincekovic, N. J. Colloid Interface Sci. 2005, 285, 342-350.

(15) Nisha, C. K.; Manorama, S. V.; Kizhakkedathu, J. N.; Maiti, S. Langmuir 2004, 20, 8468-8475.

(16) Lleres, D.; Clamme, J. P.; Dauty, E.; Blessing, T.; Krishnamoorthy, G.; Duportail, G.; Mely, Y. Langmuir 2002, 18, 10340-10347.

(17) Izumrudov, V. A.; Zhiryakova, M. V.; Goulko, A. A. Langmuir 2002, 18, 10348-10356.

(18) Melnikov, S. M.; Sergeyev, V. G.; Yoshikawa, K. J. Am. Chem. Soc. 1995, 117, 9951-9956.

(19) Costa, D.; Hansson, P.; Schneider, S.; Miguel, M. G.; Lindman, B. Biomacromolecules 2006, 7, 1090-1095.

(20) Melnikov, S. M.; Sergeyev, V. G.; Yoshikawa, K. J. Am. Chem. Soc. 1995, 117, 2401-2408.

(21) Melnikov, S. M.; Sergeyev, V. G.; Yoshikawa, K. Recent Res. Dev. Chem. Sci. 1997, 1, 69-113.

(22) Melnikov, S. M.; Sergeyev, V. G.; Yoshikawa, K.; Takahashi, H.; Hatta, I. J. Chem. Phys. 1997, 107, 6917-6924.

(23) Melnikov, S. M.; Yoshikawa, K. Biochem. Biophys. Res. Commun. 1997, 230, 514-517.

(24) Mel'nikova, Y. S.; Lindman, B. Langmuir 2000, 16, 5871-5878.

(25) Dias, R.; Mel'nikov, S.; Lindman, B.; Miguel, M. G. Langmuir 2000, 16, 9577-9583.

(26) Le Ny, A. L. M.; Lee, C. T. J. Am. Chem. Soc. 2006, 128, 64006408 .

(27) Dasgupta, A.; Das, P. K.; Dias, R. S.; Miguel, M. G.; Lindman, B.; Jadhav, V. M.; Gnanamani, M.; Maiti, S. J. Phys. Chem. B 2007, 111, $8502-8508$.

(28) Sambrook, E. F. Fritsch, E. J. Maniatis, T. Molecular Cloning: A Laboratory Manual; Cold Spring Harbor Laboratory Press: New York, 1989.

(29) Matsuzawa, Y.; Yoshikawa, K. Nucleosides Nucleotides 1994, 13, $1415-1423$.

(30) Barthel, J.; Feuerlein, F.; Neueder, R.; Wachter, R. J. Solution Chem. 1980, 9, 209-219.

(31) Evans, D. F.; Wennerström, H. The Colloidal Domain. Where Physics, Chemistry and Biology, and Technology Meet, 2nd ed.; WileyVCH: New York, 1999.

(32) Nelson, D. L.; Cox, M. M. Lenhinger Principles of Biochemistry, 3rd ed.; Wroth Publishers: New York, 2000.

(33) Holmberg, K.; Jönsson, B.; Kronberg, B.; Lindman, B. Surfactants and Polymers in Aqueous Solution, 2nd ed.; John Wiley \& Sons, Ltd: West Sussex, U.K., 2003.

(34) Carpena, P.; Aguiar, J.; Bernaola-Galvan, P.; Ruiz, C. C. Langmuir 2002, 18, 6054-6058.

(35) Wallin, T.; Linse, P. Langmuir 1996, 12, 305-314.

(36) Hayakawa, K.; Santerre, J. P.; Kwak, J. C. T. Biophys. Chem. 1983, $17,175-181$.

(37) Inman, R. B.; Jordan, D. O. Biochim. Biophys. Acta 1960, 42, 421426.

(38) Korolev, N. I.; Vlasov, A. P.; Kuznetsov, I. A. Biopolymers 1994, $34,1275-1290$. 
(39) Rosa, M.; Dias, R.; Miguel, M. D.; Lindman, B. Biomacromolecules 2005, 6, 2164-2171.

(40) Costa, D.; Miguel, M. G.; Lindman, B. J. Phys. Chem. B 2007, 111, 10886-10896.

(41) Merta, J.; Stenius, P. Colloids Surf. A 1997, 122, 243-255.

(42) Merta, J.; Torkkeli, M.; Ikonen, T.; Serimaa, R.; Stenius, P. Macromolecules 2001, 34, 2937-2946.

(43) Starodoubtsev, S. G.; Khokhlov, A. R.; Sokolov, E. L.; Chu, B. Macromolecules 1995, 28, 3930-3936.

(44) Norrman, J.; Lynch, I.; Piculell, L. J. Phys. Chem. B 2007, 111, 8402-8410.

(45) Wallin, T.; Linse, P. J. Phys. Chem. 1996, 100, 17873-17880.
(46) Dias, R. S.; Miguel, M. G.; Lindman, B. In DNA Interactions with Surfactants and Polymers; Dias, R. S., Lindman, B., Eds.; John Wiley \& Sons: Hoboken, NJ, 2008, p 367.

(47) Dias, R. S.; Svingen, R.; Gustavsson, B.; Lindman, B.; Miguel, M. G.; Akerman, B. Electrophoresis 2005, 26, 2908-2917.

(48) Guldbrand, L.; Jönsson, B.; Wennerström, H.; Linse, P. J. Chem. Phys. 1984, 80, 2221-2228.

(49) Khan, M. O.; Mel'nikov, S. M.; Jönsson, B. Macromolecules 1999, $32,8836-8840$.

(50) Mel'nikov, S. M.; Khan, M. O.; Lindman, B.; Jönsson, B. J. Am. Chem. Soc. 1999, 121, 1130-1136.

JP8027935 